\title{
Estabilización de suelos expansivos con ceniza de mazorca de maíz en la ciudad del Cusco
}

\section{Stabilization of expansive soils with corncob ash in the city of Cusco}

Dante Quispe Vilca ${ }^{1}$

${ }^{1}$ Pontificia Universidad Católica del Perú

Correoelectrónico:Dante.quispev@pucp.edu.pe

\section{Resumen}

La explotación de canteras para mejorar suelos en carreteras ha ido incrementando a lo largo de los años. Este problema genera la depredación de ecosistemas tanto en cerros como en ríos. En este sentido, el desarrollo de este estudio busca analizar el comportamiento físico y químico de suelos expansivos con ceniza de mazorca de maíz, el cual es un producto abundante y usualmente desechado en la ciudad del Cusco. El proyecto está divido en cuatro etapas. En la primera etapa se realizó una revisión de bibliográfica acerca de suelos expansivos y su mejoramiento mediante estabilización química con diferentes productos. Luego, en la segunda etapa, se realizó la extracción de la muestra en campo. En la tercera etapa, se realizó ensayos de mecánica de suelos al suelo en estudio sin ceniza y con diferentes cantidades de ceniza de mazorca de maíz. Por último, en la cuarta etapa se analizó los resultados mediante gráficas y se encontró la proporción óptima para mejorar este tipo de suelos con ceniza de mazorca de maíz. Asimismo, en esta etapa se comparó los resultados obtenidos con otros estudios similares en otros lugares. Entre las principales mejoras del suelo, se puede mencionar la reducción de la plasticidad en $42 \%$ para un porcentaje de ceniza de $10 \%$. Asimismo, también se mejoró la capacidad portante del suelo CBR en un $62 \%$ para un porcentaje de ceniza de $8 \%$. Por lo tanto, se puede concluir que un suelo expansivo, usualmente reemplazado por suelos granulares de cantera, puede ser mejorado con la adición de ceniza de mazorca de maíz sin perjudicar los ecosistemas en los lechos de ríos y cerros.

Palabras clave: Estabilización de suelos, suelos expansivos, suelos arcillosos, estabilización química con cenizas, mejoramiento de suelos 


\begin{abstract}
Quarrying to improve road soils has been increasing over the years. This problem generates those ecosystems are depleted both in hills and in rivers. In this sense, the development of this study seeks to analyze the physical and chemical behavior of expansive soils with corn cob ash, which is an abundant and usually discarded product in the city of Cusco. The project is divided into four stages: in the first stage, a literature review was carried out on expansive soils and their improvement through chemical stabilization with different products. Then, in the second stage, the sample was extracted from the earth. In the third stage, soil mechanics tests were carried out on the study soil without ash and with different amounts of corn cob ash. Finally, in the fourth stage, the results were analyzed using graphs and the optimal proportion was found to improve this type of soils with corn cob ash. Among the main improvements of the soil, it is possible to mention the reduction of plasticity by $42 \%$ for an ash percentage of $10 \%$. Likewise, the bearing capacity of the CBR soil was also improved by $62 \%$ for an ash percentage of $8 \%$. Therefore, it can be concluded that an expansive soil, usually replaced by granular quarry soils, can be improved with the addition of corn cob ash without damaging the ecosystems in the river beds and hills.
\end{abstract}

Keywords: Soil stabilization, expansive soils, clay, ash chemical stabilization, soil improvement

\title{
Introducción
}

El mejoramiento de suelos expansivos se ha realizado a lo largo de los años reemplazando estos suelos por suelos granulares más resistentes. Sin embargo, este método causaba la depredación de lechos de río o canteras afectando su ecosistema. Debido a este resultado, hoy en día se sugiere mejorar los suelos expansivos con algún agente químico natural que permita reducir la plasticidad de estos suelos y mejorar su capacidad portante.

El suelo estudiado fue un suelo extraído de la vía expresa de la ciudad del Cusco. El suelo fue una arcilla de baja plasticidad (CL). Este suelo es el que más se presenta en toda la extensión de la vía expresa de la ciudad del Cusco. En la construcción de esta vía se eligió mejorar este suelo por reemplazo de material de canteras de diferentes lugares y como resultado de esto se está depredando más de $100,000 \mathrm{~m}^{2}$ de extensión de terreno (Enriquez, 2019). En el mapa $\mathrm{N}^{\circ} 1$ y en la figura $\mathrm{N}^{\circ} 1$ se muestra la localización del lugar donde se extrajo las muestras para su análisis.

El presente estudio busca analizar las propiedades físicas y mecánicas del suelo expansivo encontrado en la vía expresa de la Ciudad del Cusco agregando diferentes cantidades de ceniza de mazorca de maíz para luego encontrar el óptimo porcentaje de ceniza a agregar a este tipo de suelos. 


\section{Mapa 1}

Mapa de ubicación del lugar de la muestra analizada.
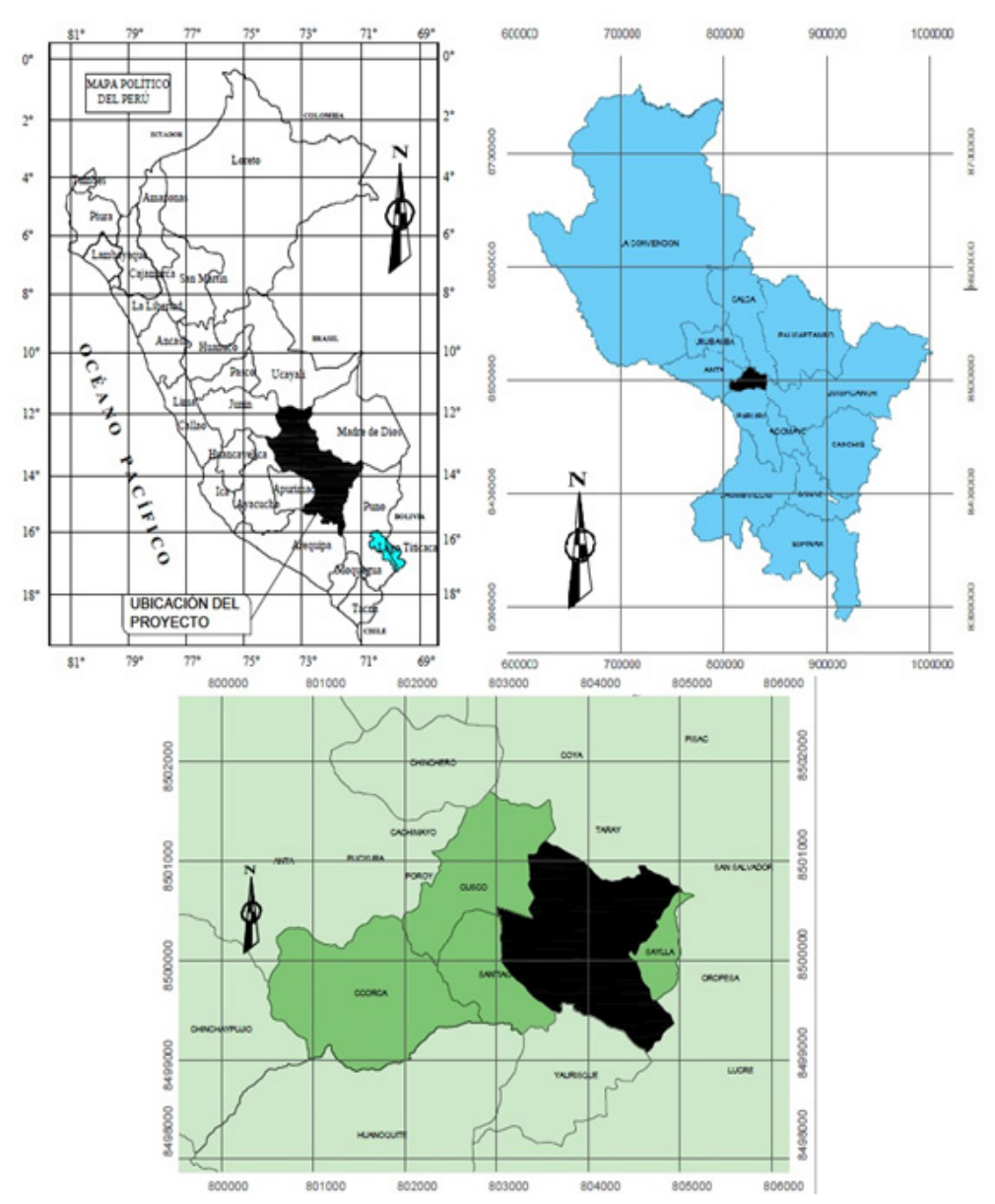

Fuente: http://sigmed.minedu.gob.pe/descargas/, (13/09/2021)

\section{Figura 1}

\section{Ubicación satelital de la muestra analizada}

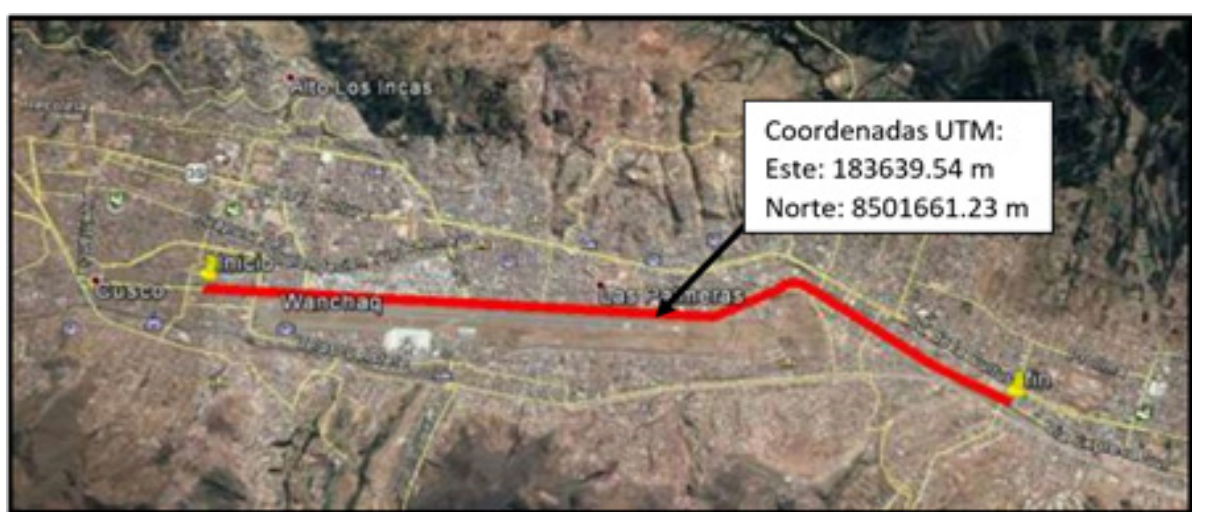

Fuente: Google Earth, (13/09/2021) 


\section{Suelos y tipos de suelos}

Según Budhu, los suelos son materiales que provienen de la erosión de las rocas (Budhu,2015). En efecto, los suelos provienen de la desintegración de las rocas; por ello, las propiedades de estos dependen de los minerales presentes en las rocas.

En el campo de la geotecnia, los suelos pueden dividirse en suelos granulares y suelos cohesivos. Los primeros pueden dividirse a su vez en gravas y arenas, mientras que los segundos pueden dividirse en limos y arcillas. A lo largo de los años, se desarrollaron varios tipos de clasificación. El más usado a nivel mundial y el que se usará en el presente artículo es la clasificación SUCS, el cual fue creado por el American Society for Testing and Materials ASTM. Acorde con esta clasificación, los suelos finos son los que tienen partículas que pasan más del 50\% la malla \#200 $(0.075 \mathrm{~mm})$, mientras que los suelos granulares son los que retienen más del $50 \%$ la malla \#200 (DAS, 2015)

\section{Suelos expansivos y su plasticidad}

Los suelos expansivos son suelos que están formados por partículas inferiores a la malla \#200 y además presentan un índice de plasticidad de 11 o más (Juárez \& Rico, 2007). En la construcción de caminos de vecinales, estos suelos son muy desfavorables, puesto que al estar saturados disminuyen radicalmente su capacidad de soporte y se erosionan rápidamente con el paso de los vehículos.

La plasticidad es una propiedad índice fundamental de los suelos cohesivos, la cual se expresa como la facilidad de moldearse de un suelo cuando presenta cierto contenido de humedad. Según Atterberg, un suelo cohesivo puede ser tan consistente como un ladrillo o un lodo semilíquido con un determinado contenido de agua (Terzagui, 1996). Entre estos dos extremos el suelo se presenta en un estado plástico en el cual puede ser moldeado con facilidad.

Atterberg también presenta un método para medir está plasticidad, el cual es representado por el Índice de plasticidad, el cual se calcula como la diferencia entre el límite líquido y el límite plástico del suelo. El límite líquido es el contenido de humedad en el cual el suelo deja de estar en estado semilíquido y empieza a estar en estado plástico, mientras que el límite plástico es el contenido de humedad en el cual el suelo deja de estar en estado plástico y comienza a tornarse semisólido. (Juárez \& Rico, 2007).

\section{Capacidad de soporte CBR}

La capacidad de soporte de un suelo compactado se mide a través del valor de $\mathrm{CBR}$, el cual es un valor de resistencia comparado con el de la piedra de California expresado en porcentaje. Este valor se puede presentar para el $95 \%$ o el $100 \%$ de la máxima densidad seca del suelo dependiendo de la capa que se está analizando. En pavimentos, el valor de CBR sirve para valorar la calidad del suelo analizado. Según el manual de suelos y pavimentos del MTC, la subrasante se puede clasificar según sus valores de CBR como se indica en la tabla 1. 
Tabla 1

Categorías de la subrasante según el valor de CBR

\begin{tabular}{|c|c|}
\hline Categorías de Subrasante & CBR \\
\hline So: Subrasante Inadecuada & $\mathrm{CBR}<3 \%$ \\
\hline $\mathrm{S}_{1}$ : Subrasante Pobre & $\begin{array}{c}\text { De CBR } \geq 3 \% \\
\text { A CBR }<6 \%\end{array}$ \\
\hline $\mathrm{S}_{2}$ : Subrasante Regular & $\begin{array}{l}\mathrm{De} C B R \geq 6 \% \\
\mathrm{~A} C B R<10 \%\end{array}$ \\
\hline $\mathrm{S}_{3}:$ Subrasante Buena & $\begin{array}{c}\text { De CBR } \geq 10 \% \\
\text { A CBR }<20 \%\end{array}$ \\
\hline $\mathrm{S}_{4}$ : Subrasante Muy Buena & $\begin{array}{c}\text { De CBR } \geq 20 \% \\
\text { A CBR }<30 \%\end{array}$ \\
\hline S5: Subrasante Excelente & $\mathrm{CBR} \geq 30 \%$ \\
\hline
\end{tabular}

Fuente: MTC (2014)

\section{Estabilización de suelos}

Según el manual de construcción de carreteras, la estabilización de los suelos es el mejoramiento de las propiedades mecánicas de un suelo a través de procesos físicos o químicos (MTC, 2013). En primer lugar, la estabilización física consiste en la adición de productos que no generen una reacción química, pero que mejoren la resistencia del suelo compactado. Estos materiales pueden ser suelos granulares o geosintéticos. En segundo lugar, la estabilización química consiste en mejorar el suelo arcilloso con la adición de productos químicos que permitan cambiar las propiedades índices del suelo. Productos como la cal o la ceniza son los principales estabilizadores químicos.

Mediante la estabilización se suelos se pretende principalmente incrementar el valor de CBR y reducir la plasticidad de un determinado suelo. Sobre esto, Huang propone mejorar los suelos cuando estos presentan un valor de CBR menor a $6 \%$ o el índice de plasticidad del suelo es mayor a 10 (Huang, 2010).

\section{Estabilización con ceniza de mazorca de maíz}

La mazorca de maíz es usualmente considerada como un desperdicio y tiene como disposición final los botaderos de basura. Sin embargo, al quemarse este material, se convierte en ceniza, la cual puede utilizarse como un estabilizador químico de los suelos arcillosos. Según Castro, las cenizas contienen un alto contenido de sílice, el cual al ser mezclado con el calcio presente en el suelo produce silicatos de calcio (Castro, 2018). Estos silicatos de calcio de manifiestan en una reducción del índice de plasticidad y el aumento de la capacidad de soporte (CBR). Sin embargo, una gran proporción de ceniza puede, por el contrario, reducir el valor de CBR de la subrasante. 


\section{Metodologia}

La metodología del presente trabajo es una metodología cuantitativa experimental, la cual incluye cuatro etapas. En la primera etapa se realizó una revisión de la bibliografía de estudios e investigaciones similares. Luego, en la segunda etapa, se realizó la extracción de la muestra en campo. En la tercera, se aplicó la ceniza de mazorca de maíz en diferentes proporciones a un suelo arcilloso y se ejecutó diferentes ensayos de suelos. Por último, en la cuarta se analizó los resultados de los diferentes ensayos de suelos y obtuvo la cantidad adecuada de ceniza de mazorca de maíz para estabilizar un suelo arcilloso. En esta etapa también se comparó los resultados con otros estudios similares en otros lugares del mundo.

\section{Figura 2}

Metodología del mejoramiento de suelos

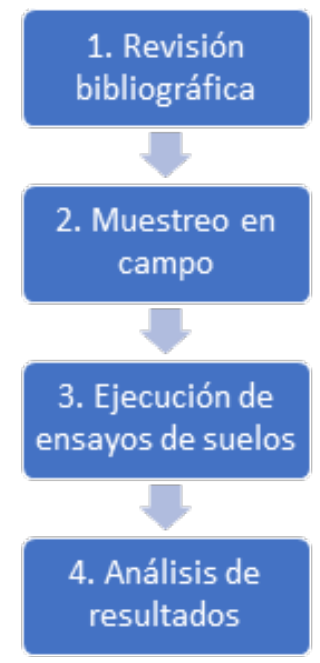

Fuente: Propia

\section{Muestreo en campo}

Luego de realizar la revisión bibliográfica relacionada a estabilización de suelos arcillosos, se procedió a buscar una muestra de suelo arcilloso en la ciudad del Cusco. Para ubicar esta muestra se usó el perfil estratigráfico de la vía expresa de la ciudad del Cusco, la cual se muestra en la figura 3. En este gráfico se puede apreciar que desde la progresiva $0+820$ hasta la progresiva $2+000$ el suelo predominante es una arcilla de baja plasticidad CL a profundidades mayores a $1 \mathrm{~m}$. Por lo tanto, la muestra para los estudios se extrajo a $1.50 \mathrm{~m}$ de la progresiva $1+200$, la cual tiene las siguientes coordenadas: Este $183639.54 \mathrm{~m}$ y Norte $8501661.23 \mathrm{~m}$. 


\section{Figura 3}

Perfil estratigráfico de la vía expresa de la ciudad del Cusco

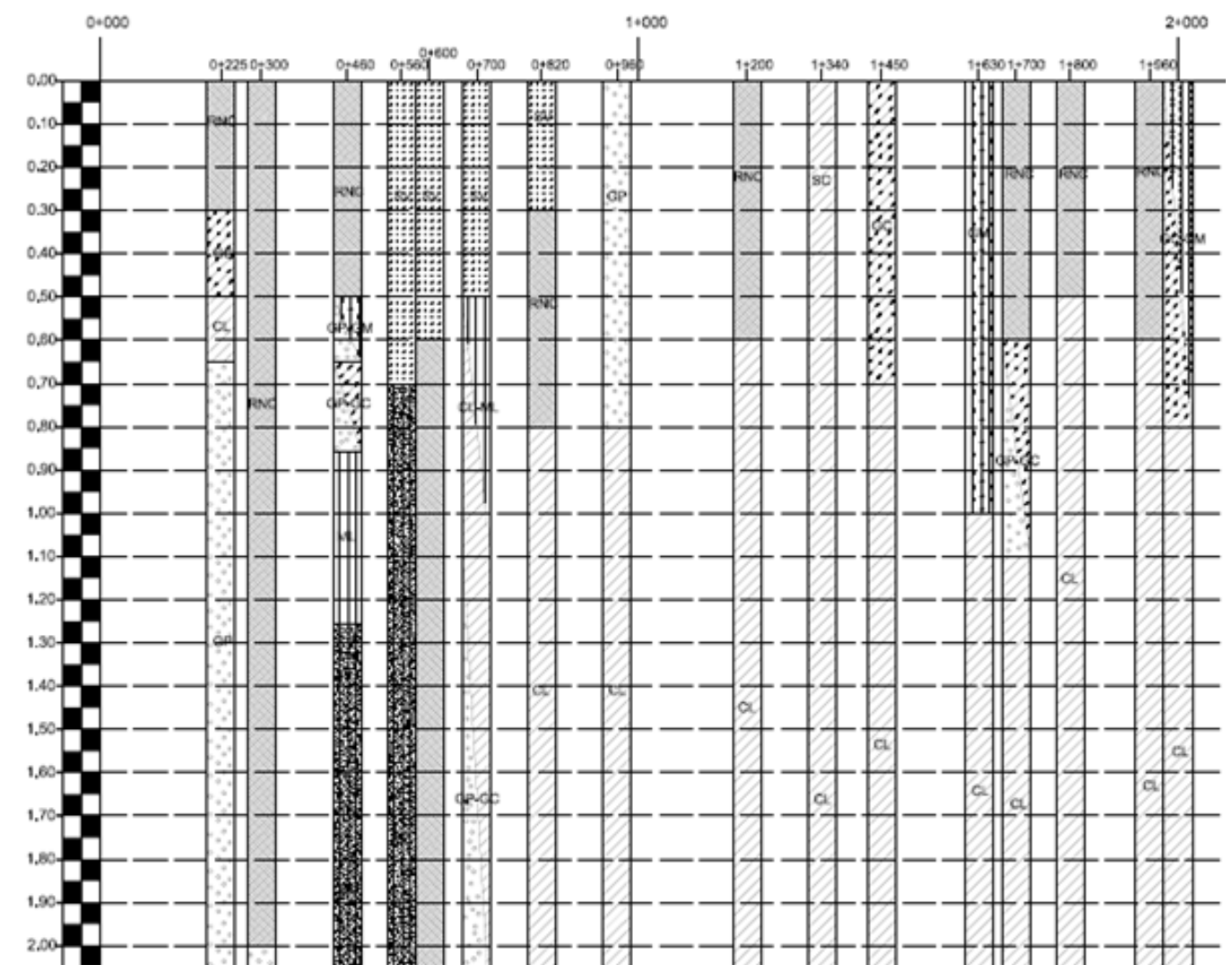

Fuente:(Enriquez, 2019)

Los criterios de inclusión utilizados fueron los siguientes:

-Suelos con índice de plasticidad IP mayor a 10\%.

-Suelos con porcentaje de finos mayor a 50\%

Los criterios de exclusión utilizados fueron los siguientes:

-Suelos con índice de plasticidad IP menor a 10\%.

-Suelos con porcentaje de finos menor a $50 \%$

\section{Resultados}

En este acápite se muestra y se analiza los resultados de los ensayos de suelos efectuados al suelo en estudio con diferentes cantidades de ceniza de mazorca de maíz $(0 \%, 2 \%, 4 \%, 6 \%, 8 \%$ y $10 \%)$. Los ensayos efectuados a este suelo fueron los ensayos de granulometría, límite líquido, límite plástico, proctor modificado y CBR. Sin embargo, los más relevantes para observar la mejora del suelo fueron los ensayos de límite líquido, límite plástico y CBR de 0.1 " al $100 \%$ de la máxima densidad seca, ya que estos permiten ver como se redujo la plasticidad y aumentó la resistencia a las cargas que circulen por dicho pavimento. 
Con respecto al límite líquido, este tuvo un valor de 31.7\% para el suelo sin ninguna adición de ceniza. Luego, mientras más se agregó ceniza esté disminuyo levemente hasta $30.1 \%$ cuando el porcentaje de ceniza fue de $10 \%$. El valor de límite plástico también aumento ligeramente de $21.56 \%$ de suelo sin ceniza a $24.23 \%$ de suelo con 10\% de ceniza. En la figura 4 se puede apreciar los valores de límite liquido plástico para diferentes proporciones de ceniza.

\section{Figura 4}

\section{Límite líquido y plástico a diferentes cantidades de ceniza}

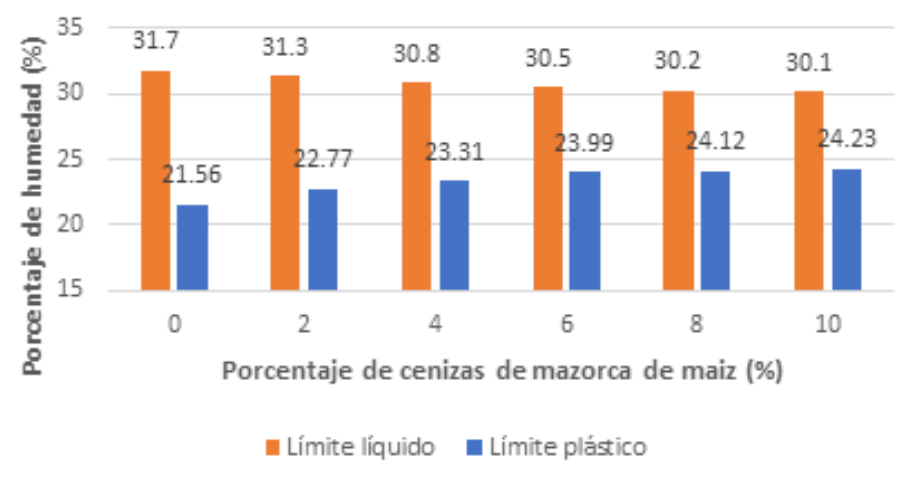

Fuente: Propia

Por otro lado, en consecuencia, del aumento del valor de límite líquido y la disminución del límite plástico, el índice de plasticidad se redujo considerablemente de $10.14 \%$ para suelo natural a $5.87 \%$ para suelo con $10 \%$ de ceniza. Esta reducción de plasticidad se produjo debido a la adición de ceniza, el cual es un producto no plástico. En la figura 5 se puede mostrar la disminución del índice de plasticidad para diferentes porcentajes de ceniza de mazorca de maíz.

\section{Figura 5}

Índice de plasticidad a diferentes cantidades de ceniza

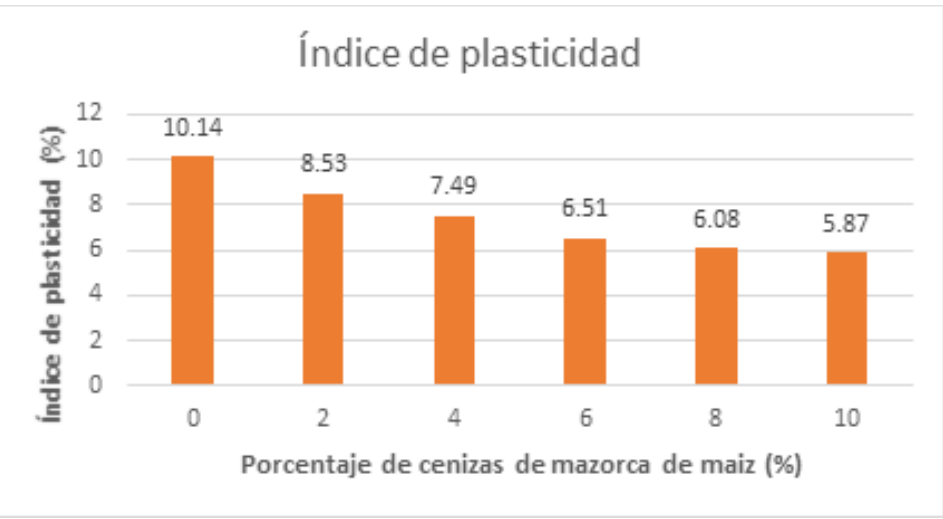


Por último, en la figura 6, el valor de CBR aumentó considerablemente de 7.2\% para el suelo sin ceniza a $19.1 \%$ para el suelo con $8 \%$ de ceniza. Sin embargo, luego de agregar más ceniza (10\%) el valor de CBR comenzó a disminuir, lo cual indica que el valor óptimo de ceniza de mazorca de maíz es de $8 \%$. El aumento inicial de CBR se debe a que, al reducir su plasticidad, el suelo empezó a tornarse más granular; por lo tanto, mejoraron sus propiedades mecánicas. No obstante, después de cierta de cantidad de ceniza, el valor de CBR comenzó a disminuir debido a que el suelo tiene un límite para reaccionar con la ceniza. Por encima de este valor, la ceniza ya no aporta a las propiedades mecánicas del suelo, entonces el valor de resistencia la corte diminuye.

\section{Figura 6}

CBR a diferentes cantidades de ceniza

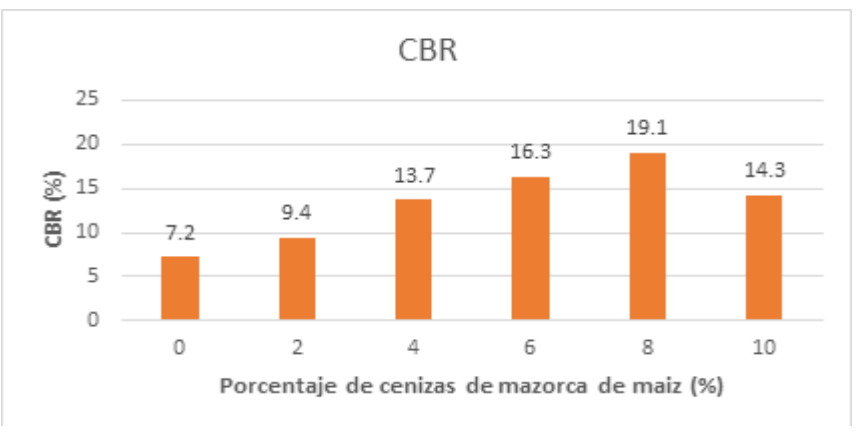

Fuente: Propia

\section{Discusión}

En este acápite se comparó los resultados obtenidos de la estabilización de los suelos con ceniza de mazorca de maíz con los resultados de la estabilización de cenizas de cascara de arroz de otros dos investigadores. En la tabla 2 se muestra la comparación de las principales propiedades del suelo como su uso en obras viales.

\section{Tabla 2}

Resumen de las propiedades físicas y mecánicas de suelos naturales y suelos estabilizados con diferentes tipos de cenizas a diferentes porcentajes.

\begin{tabular}{|c|c|c|c|c|c|c|}
\hline \multirow{2}{*}{$\begin{array}{c}\text { Resultados de } \\
\text { ensay os }\end{array}$} & \multicolumn{2}{|c|}{$\begin{array}{c}\text { Ceniza de mazorca de maiz } \\
\text { (presente investigación) }\end{array}$} & $\begin{array}{c}\text { Ceniza de cáscara de arroz } \\
\text { (Lamogą 2017) }\end{array}$ & $\begin{array}{c}\text { Ceniza de cáscara de arroz } \\
\text { (Kumar, 2017) }\end{array}$ \\
\cline { 2 - 8 } & Sin ceniza & Con ceniza (8\%) & Sin ceniza & Con ceniza (7\%) & Sin ceniza & Conceniza (7.5\%) \\
\hline Ĺmite líquido (\%) & 31.7 & 30.2 & 44.53 & 27.51 & 36.1 & 34.5 \\
\hline Límite plástico (\%) & 21.56 & 24.12 & 24.51 & 19.38 & 23.7 & 28.3 \\
\hline Índice plástico (\%) & 10.14 & 6.08 & 20.02 & 8.14 & 12.36 & 8.55 \\
\hline CBR (\%) & 7.2 & 19.1 & 2.85 & 7.8 & 6.3 & 18.83 \\
\hline
\end{tabular}

Fuente: Propia 
En general, en los tres casos, el porcentaje óptimo de ceniza fue en promedio $7.5 \%$. También se pudo observar una reducción del límite líquido. Sin embargo, el límite plástico solo se redujo en para el estudio realizado por Llamoga. A pesar de ello, el índice plástico disminuyó en los tres estudios. Finalmente, el valor de la relación de soporte California (CBR) aumentó en las tres investigaciones comprobando así el mejoramiento de suelos con el uso de cenizas.

Con respecto a las propiedades físicas del suelo, los resultados de la presente investigación muestran una reducción porcentual de 3\% aproximadamente, la cual es similar a la del estudio de Kumar. De igual forma, el límite plástico aumentó en una proporción cerca de $14 \%$ con la adición de ceniza de mazorca de maíz al $8 \%$ y con la adición de ceniza de cáscara de arroz al 7.5\%. Sin embargo, el resultado de índice plástico se parece más a los resultados de Llamoga, pues en ambos casos este valor se redujo en alrededor de $50 \%$.

Por otro lado, en las tres investigaciones se incrementa el valor de CBR. Sin embargo, estás aumentaron en diferentes magnitudes. En el presente estudio, este valor aumentó un poco que el doble de su valor inicial (de 7.2\% a 19.1\%), mientras que, en las otras estabilizaciones, el valor de CBR fue casi tres veces su valor inicial. En el estudio de Llamoga, este aumentó de $2.85 \%$ a $7.8 \%$ y en el estudio de Kumar, este incremento fue de $6.3 \%$ a $18.83 \%$. Estos resultados probablemente se deban a que la procedencia de las cenizas para este estudio (mazorca de maíz) es diferente que de los otros estudios (cáscara de arroz).

\section{Conclusiones}

El suelo analizado en el presente estudio fue una arcilla mal gradada con arena (CL) con alta plasticidad (IP=10.14) y una capacidad de soporte pobre $(\mathrm{CBR}=7.2)$. Sin embargo, al añadirle ceniza de mazorca de maíz se puedo mejorar sus propiedades tanto físicas como mecánicas. La proporción óptimas de ceniza para este suelo fue de $8 \%$. Entre las principales mejoras se puede mencionar las siguientes:

La adición de ceniza de mazorca de maíz pudo mejorar el comportamiento no plástico del suelo reduciendo el índice de plasticidad de 10.14\% 6.08\%. Es decir, reduciendo la plasticidad del suelo, la cual es nociva para el pavimento.

La colocación de ceniza de mazorca de maíz a un $8 \%$ pudo incrementar el valor de inicial de CBR (7.2\%) a más del doble de su valor (19.10\%). Siendo este el máximo valor alcanzado, ya que para cantidades mayores a $8 \%$ este valor tiende a disminuir.

\section{Recomendaciones}

Suelos similares al suelo analizado se pueden encontrar en otros lugares. Por ello, se sugiere utilizar la cantidad optima de ceniza de mazorca de maíz (8\%) para la mejora de dichos suelos. 
Se recomienda realizar estudios parecidos utilizando otros tipos de ceniza para comparar los resultados y ver qué tipo de ceniza resulta mejorar para la estabilización de suelos arcillosos.

Por último, se recomienda que, en obras viales, cuando se encuentre este tipo de suelos, realizar ensayos para analizar la probabilidad de mejorar los suelos con cenizas para evitar la extracción de grava de cuerpos de ríos o canteras de cerro.

\section{Declaración de conflicto de intereses}

El autor declara no presentar ningún tipo de conflicto de intereses. 


\section{Referencias}

Budhu, M. (2015). Soil Mechanics Fundamentals. Edited by J. Wiley and S. Ltd. Chichester: Wiley Blackwell.

Castro, A. (2017). Estabilización de suelos arcillosos con ceniza de cascara de arroz (Tesis inédita de titulación). Universidad Nacional de Ingeniería.

Das, B. (2001). Fundamentos de Ingeniería Geotécnica(1a. ed.). México: International Thomson.

Enriquez, Y. (2019). Estudio de suelos, canteras y fuentes de agua para la Vía expresa de la ciudad del. Expediente técnico del mejoramiento integral de la vía expresa de la ciudad del Cusco. Cusco.

Kumar, A., Gaurav, K., Kishor, R. \& Suman, S. (2017). Stabilization of Alluvial Soil for Subgrade Using Rice Husk Ash, Sugarcane Bagasse Ash and Cow Dung Ash for Rural Roads. International Journal of Pavement Research and Technology.

Llamoga, L. (2017). Evaluación del potencial de expansión y capacidad portante de suelos arcillosos usados en subrasantes al adicionar ceniza de cascarilla de arroz (Tesis de titulación). Universidad Nacional de Cajamarca.

Huang, Y. (1993). Pavement analysis and design. Kentucky: Prentice-Hall.

Juárez, E. \& Rico, A. (2006). Mecánica de suelos: Fundamentos de la mecánica de suelos (1a ed.). México: Limusa.

Ministerio de Educación (2021) Descarga de información espacial del MED. Recuperado de http://sigmed.minedu.gob.pe/descargas/.

Ministerio de Transportes y comunicaciones (2014) Manual de Carreteras-Suelos, Geología, Geotecnia y Pavimentos. Lima: Editora Macro.

Ministerio de Transportes y comunicaciones (2013) Manual de Carreteras: Especificaciones generales para la construcción de carreteras. Lima: Editora Macro.

Ministerio de Transportes y Comunicaciones (2016) Manual Ensayo de Materiales. Lima: Editora Macro

Terzaghi, K. \& and Ralph B. (1996). Soil Mechanics in Engineering Practice Third Edition (3rd edition). New York: john wiley \& sons. 